\title{
THE EFFECT OF OVARIECTOMY ON WOMEN'S PERIODONTAL STATUS: A PILOT STUDY
}

\author{
Kanwal Sohail, Mahrukh Zafar*, Maham Zulfiqar**, Amber Kiyani \\ Islamic International Dental College, Riphah International University Islamabad Pakistan, *Lowa State University, Ames, United State, **COMSATS University, \\ Islamabad Pakistan
}

\begin{abstract}
Objective: To determine the effect of bilateral oophorectomy on the periodontal status of otherwise healthy women when compared with normal controls.

Study Design: Mixed-method study.

Place and Duration of Study: Riphah International University, Islamabad, from Apr to Sep 2016.

Methodology: A total of 24 women with and without oophorectomy procedures were recruited. Twelve women who has undergone radical hysterectomies at least 6 months prior to the evaluation were recruited for this investigation. These women underwent a comprehensive oral examination with detailed documentation of their periodontal status following a thorough medical/dental history. An age- and sex-matched control population underwent a similar examination.

Results: Statistically significant results were noted for probing, color, consistency, texture and contour of gingiva with the $p$-value of $0.007,0.001,0.002,0.005$ and 0.037 when the results were compared with the control population.

Conclusion: Bilateral oophorectomy does not compromise the periodontal health of otherwise healthy women.
\end{abstract}

Keywords: Menopause, Oral health, Ovariectomy, Periodontitis.

This is an Open Access article distributed under the terms of the Creative Commons Attribution License (https://creativecommons.org/licenses/by-nc/4.0/), which permits unrestricted use, distribution, and reproduction in any medium, provided the original work is properly cited.

\section{INTRODUCTION}

Hysterectomy is a gynecological surgical procedure that requires removal of the uterus, occasionally ovaries, and other associated organs. Over $78 \%$ of the hysterectomy procedures in women over the age of 45 are accompanied by bilateral ovariectomy/oophorectomy. Indications for this procedure includebenign tumors, ovarian cancer, severe endometriosis, bilateral tubo-ovarian abscess, familial breast-ovarian cancer syndrome and severe premenstrual syndrome ${ }^{1}$. It is estimated that one in three women in the United States, and one in five women in the United Kingdom undergo this procedure before reaching 60 years of age ${ }^{2}$. A Indian study has reported a prevalence of $6 \%$ in women $^{3}$. Another study conducted in Gujrat, India documented a higher incidence of hysterectomies that is 20.7/1000 women- year at a mean age of 36 years ${ }^{4}$. Although baseline statistics from Pakistan are not available, hysterectomy coupled with oophorectomyis one of the most frequently performed surgical procedure in women, due to complaints of menorrhagia and fibroids 5 .

Bilateral oophorectomy results in low estrogen levels in the body. This causes temperature intolerance, depressive illnesses, increased susceptibility to urinary tract infections and weight gain. Long term deficiency

Correspondence: Ms Kanwal Sohail, H-I/1, Presidency Staff Colony, Constitutional Avenue, G-5/1 Islamabad Pakistan

Received: 27 Mar 2020; revised received: 10 May 2020; accepted: 19 May 2020 of estrogen predisposes women to cardio-vascular diseases, and Alzheimer disease ${ }^{6}$. Estrogen plays a fundamental role in skeletal growth and bone homeostasis, thus predisposing women undergoing this procedure to osteoporosis?.

The symptoms associated with oophorectomy and menopause should be similar as both result in estrogen deficiency. It is also known that menopauseassociated estrogen deficiency can result in burning mouth syndrome, xerostomia, taste alterations, mucosal changes, periodontitis, decreased bone mineral content of alveolar bone, and neurological disorders ${ }^{8}$. In addition, desquamative gingivitis can be caused by endocrinal imbalance, menopause, hysterectomy or ovariectomy ${ }^{9}$. While limited information is available about the dental implications of oophorectomy, we do know that post-menopausal women are 4 times more likely to develop osteoporosis leading to systemic and local bone loss than men ${ }^{10}$. Estrogen levels are in a tight balance; higher levels can cause gingivitis while low levels potentiate alveolar bone loss leading to periodontitis 7 . Hormone replacement therapy helps in preserving the oral health in estrogen deficient patients ${ }^{11}$.

This creates a need for dentists to be aware of the dental implications of estrogen deficiency in females. Since little is known about the estrogen-deficient state as a result of bilateral oophorectomy, this study was planned to evaluate the effects of this procedure on the periodontal health of otherwise healthy women. 


\section{METHODOLOGY}

A mixed-method study was designed to study the effects of bilateral oophorectomy on oral health. This study was approved by the Ethical Review Board at Riphah International University (ref. no. IIDC/IRC/ 2019/10/003), prior to data collection from April to September 2016. Since no figures were available from Pakistan, we took the maximum prevalence of hysterectomy from an Indian study; $6 \%^{3}$. This was coupled with a confidence interval of $95 \%$ and a margin error of $5 \%$ rendering a sample size of 45 . We set our benchmark for the pilot investigation for 15 . However, it was difficult finding participants that satisfied our inclusion criteria so were forced to settle for 12 .

Twelve consenting females were enrolled in the study group through a convenience sampling technique. Inclusion criteria included pre-menopausal women who had undergone oophorectomy procedures between the range of 6 months to 10 years. Maintenance of good oral hygiene through regular brushing, flossing and routine dental visits was also required. Patients over the age of 45 , women taking hormone replacement therapy and poor oral hygiene status were excluded from the sample. All patients with significant medical histories were also excluded to minimize confounding factors. The control population consisted of age and sex-matched females who had not undergone oophorectomies.

Medical and dental history was taken from the sample population. Oral hygiene habits were inquired and preliminary oral hygiene status was assessed. Current oral symptoms, if any, color, consistency, texture and contour of gingiva, plaque index, bleeding on probing, pocket depth (CPITN), calculus (OHI-S), presence or absence of plaque retentive factors, gingival recession, furcation involvement, tooth mobility, clinically visible bone loss and xerostomia were recorded during the clinical oral examination. For detail scoring /coding of indices as shown in table-I. For evaluation of clinical bone loss, measurements were taken from cemento-enamel junction to alveolar bone crest.

Data was tabulated and analyzed using SPSS-22. The $p$-value $\leq 0.05$ was considered significant at $95 \%$ confidence interval. Descriptive analysis was done and Fisher's exact test was applied for all the parameters under study for comparison between the two groups.

\section{RESULTS}

This pilot studyincluded a total of 24 females with the mean age of $38.6 \pm 3.4$ year. Twelve $(50 \%)$ women had undergone bilateral at least 6 prior to this visit. Twelve (50\%) healthy women were taken as healthy controls. Following the coding used as per proforma, the only parameters that showed statistically significant difference between study group and control group were bleeding on probing, color, consistency, texture and contour of gingiva with the $p$-value of $0.007,0.001,0.002,0.005$ and 0.037 respectively (table).

\section{DISCUSSION}

To the best of our knowledge, human studies on the effects of surgically induced estrogen deficiency, or oophorectomy on oral health are deficient in the literature. However, a few animal studies show an association between the estrogen deficiency and periodontal health. Most of these studies include experimentation on rats, as the structure of periodontal tissues of rats and humans are similar. The experimentally induced skeletal changes as a result of oophorectomy in rats are similar to those seen in post-menopausal women ${ }^{12}$.

Amadei et al, documented that experimentally induced periodontitis is observed in ovariectimized (OVX) rats after 90 days indicating that long-term estrogen deficiency results in the ligature induced alveolar bone loss ${ }^{13}$. Our results however do not support this notion. We were unable to identify statistically significant differences in terms of periodontal health and clinical bone loss. Only non-specific parameters like bleeding on probing, color, consistency, texture and contour of gingiva showed statistically significant difference between our sample and control groups. Since these parameters do not support a definitive diagnosis of periodontal disease, they are only considered as oral alterations of estrogen deficiency. Therefore making a strong association between oophorectomy and oral health status was difficult to determine.

In accordance with our results, a study conducted by Anbinder et al, in 2006 performed radiographic and macroscopic analysis in OVX rats. This study did not find a correlation of estrogen deficiency and periodontal health status ${ }^{14}$. Our results werealso consistent with a study done by Marques et al. They conducted a histometric evaluation of the furcation region in lower molars andfound no significant bone loss. Similarly, Orrico et al, showed no difference between OVX rats and control group in periodontal bone loss based on radiographic and densitometric analysis ${ }^{15,16}$

In contrast to our results, arat experimentation conducted by Xin-Chen et al. Demonstrated that oophorectomy resulted in the deterioration of the alveolar bone microarchitecture, alveolar crest height reduction, 
Table: Different parameters under study.

\begin{tabular}{|c|c|c|c|c|}
\hline \multicolumn{2}{|r|}{ Variables (Code) } & Case, $\mathrm{n}(\%)$ & Controls, n (\%) & $p$-value \\
\hline \multirow{4}{*}{ Cpitn } & No pocket $(0)$ & $3(25.0)$ & $7(58.3)$ & \multirow{4}{*}{0.105} \\
\hline & No pocket <3mm (1) & $5(41.7)$ & $5(41.7)$ & \\
\hline & Pocket $4-5 \mathrm{~mm}(2)$ & $4(33.3)$ & - & \\
\hline & Pocket $>6 \mathrm{~mm}(3)$ & - & - & \\
\hline \multirow{4}{*}{ Plaque Index } & No calculus $(0)$ & $2(16.7)$ & $3(25.0)$ & \multirow{4}{*}{0.397} \\
\hline & Supra2 not more than $1 / 3$ rd tooth surface0 (1) & $5(41.7)$ & $8(66.7)$ & \\
\hline & Supra $01 / 3$ rd to $2 / 3$ rd or isolated flecks of sub. (2) & $4(33.3)$ & $1(8.3)$ & \\
\hline & Supra on entire tooth or heavy band of sub. (3) & $1(8.3)$ & - & \\
\hline \multirow{4}{*}{ Gingival Recession } & No extension to MG junction (0) & $7(58.3)$ & $10(83.3)$ & \multirow{4}{*}{0.461} \\
\hline & Extension to or beyond MG junction (1) & $3(25.0)$ & $2(16.7)$ & \\
\hline & Bone or soft tissue loss present, partial root coverage (2) & $2(16.7)$ & - & \\
\hline & With gross flattening, no root coverage (3) & - & - & \\
\hline \multirow{4}{*}{ Furcation Involvement } & no clinical furcation $(0)$ & $8(66.7)$ & $11(91.7)$ & \multirow{4}{*}{0.317} \\
\hline & Bone loss up to $1 / 3$ rd width (1) & $4(33.3)$ & $1(8.3)$ & \\
\hline & Bone loss up to $2 / 3$ rd width (2) & - & - & \\
\hline & Through and through defect (3) & - & - & \\
\hline \multirow{4}{*}{ Mobility } & Normal $<1 \mathrm{~mm}(0)$ & $10(83.3)$ & $11(91.7)$ & \multirow{4}{*}{1.000} \\
\hline & Up to $1 \mathrm{~mm}$ horizontal (1) & $2(16.7)$ & $1(8.3)$ & \\
\hline & Greater than 1mm horizontal (2) & - & - & \\
\hline & greater than $2 \mathrm{~mm}$ horizontal or vertical (3) & - & - & \\
\hline \multirow{5}{*}{ Bleeding on Probing } & No bleeding $(0)$ & $10(83.3)$ & $6(50.0)$ & \multirow{5}{*}{0.007} \\
\hline & Tenous red line along gingival sulcus (1) & - & $6(50.0)$ & \\
\hline & Profuse bleeding (2) & - & - & \\
\hline & Delayed Bleeding (30-60sec) (3) & $2(16.7)$ & - & \\
\hline & Spontaneous bleeding (w/o probing) (4) & - & - & \\
\hline \multirow{2}{*}{ Xerostomia } & Absent & $4(33.3)$ & $6(50.0)$ & \multirow{2}{*}{0.680} \\
\hline & Present & $8(66.7)$ & $6(50.0)$ & \\
\hline \multirow{2}{*}{$\begin{array}{l}\text { Burning Mouth } \\
\text { Syndrome }\end{array}$} & Absent & $10(83.3)$ & $12(100.0)$ & \multirow{2}{*}{0.478} \\
\hline & Present & $2(16.7)$ & - & \\
\hline \multirow[t]{2}{*}{ Clinical Bone Loss } & Absent & $10(83.3)$ & $12(100.0)$ & \multirow{2}{*}{0.478} \\
\hline & Present & $2(16.7)$ & - & \\
\hline \multirow{3}{*}{ Color of Gingiva } & Normal color & $2(16.7)$ & $12(100.0)$ & \multirow{3}{*}{0.001} \\
\hline & Inflamed red & $10(83.3)$ & - & \\
\hline & Pale gingiva & - & - & \\
\hline \multirow[t]{3}{*}{ Consistency of Gingiva } & Normal firm and resilient & $6(50.0)$ & $7(58.3)$ & \multirow{3}{*}{0.002} \\
\hline & Edematous & - & $5(41.7)$ & \\
\hline & Fibrotic & $6(50.0)$ & - & \\
\hline & Stippled attached gingiva \& interdental papilla & - & $7(58.3)$ & \\
\hline & Loss of stippling: & $12(100.0)$ & $5(41.7)$ & \\
\hline Texture of gingiva & Peeling of surface (desquamative) & - & - & 0.005 \\
\hline & Leathery (hyperkeratotic) & - & - & \\
\hline & Nodular (drug induced enlargement) & - & - & \\
\hline & Normal scalloped & $7(58.3)$ & $12(100.0)$ & \\
\hline & Gingival enlargement & - & - & \\
\hline Contour of gingiva & McCall's Festoon (recession) & $5(41.7)$ & - & 0.037 \\
\hline & Stillman's cleft (puffy swollen) & - & - & \\
\hline
\end{tabular}

decline in the bone formation rate and increased osteoclast activity ${ }^{17}$. Similarly, Duarte et al, reported a higher inter-radicular bone loss in OVX rats. They also found that estrogen replacement or calcitonin therapy cannot protect against this bone loss ${ }^{18}$. A three dimensional micro-computed tomographic analysis revealed a combination of experimental periodontitis and oophorectomy induced systemic bone loss particularly in mandible and femoral region ${ }^{19}$. These results were verified by Dai et al, and Liu et al, studies ${ }^{20,21}$. In another OVX rat study, Romualdo et al, demonstrated the elevation of interleukin 1 beta, tumor necrosis factor alpha, interleukin 6, MMP-8, and MMP-13 in apical periodontitis. They concluding that hypoestrogenic state exacerbates inflammation and degradation of extracellular matrix components leading to development 
of apical periodontitis ${ }^{22}$. A study done by Andriy and Inna in 2019 also showed progression of inflammatory and destructive process in the periodontium worsens within 3-5 years after total ovariectomy ${ }^{23}$. A review determined that estrogen effects the normal bone mass and density. It also found that estrogen levels lead to loss of keratin, thinning of gingival tissues, redness, soreness and decreased salivary gland function ${ }^{24}$. Limited work is done on this topic warrants further research to facilitate such women in their dental problems.

The limitations of our study include asmall sample size, lack of radiographs for evaluation of bone loss and no follow ups. Future recommendations include a larger sample size accompanied by radiographic analysis to assess changes in bone. Longitudinal studies on human subjects rather than experimental rat models can allow us to compare periodontal health of women before and after the oophorectomy procedure. This can help us ascertain the effect of the procedure better. If we find that such a correlation does exist, patients who have undergone oophorectomy should be advised to maintain good oral hygiene and referred to endocrinologist for hormone replacement therapy (HRT) to maintain their estrogen levels in blood. Regular visits to dentist should also be observed.

\section{CONCLUSION}

There was no statistically significant difference between medically OVX human subjects and control group regarding oral health status. Hence this study concluded that medically OVX human subjects do not show signs of periodontitis or alveolar bone loss after 6 months of the procedure.

\section{CONFLICT OF INTEREST}

This study has no conflict of interest to be declared by any author.

\section{REFERENCES}

1. Hendrix SL. Bilateral oophorectomy and premature menopause. Am J Med 2005; 118(Suppl-12B): 131-35.

2. Al-Kadri HM, Al-Turki HA, Saleh AM. Short and long term complications of abdominal and vaginal hysterectomy for benign disease. Saudi Med J 2002; 23(7): 806-10.

3. Shekhar C, Paswan B, Singh A. Prevalence, sociodemographic determinants and self-reported reasons for hysterectomy in India. Reprod Health 2019; 16(1): 118-22.

4. Desai S, Campbell OM, Sinha T, Mahal A, Cousens S. Incidence and determinants of hysterectomy in a low-income setting in Gujarat, India. Health Policy Plan 2017; 32(1): 68-78.

5. Majeed T AR, Mahmood Z, Mahmood H. Audit of Gynaecolo- gical Hysterectomies. Pak J Med Health Sci 2013; 7(3): 684-87.

6. Friedlander $\mathrm{AH}$. The physiology, medical management and oral implications of menopause. J Am Dent Assoc 2002; 133(1): 73-81.

7. Robinson JL, Johnson PM, Kister K, Yin MT, Chen J, Wadhwa S. Estrogen signaling impacts temporomandibular joint and periodontal disease pathology. Odontol 2020; 108(2): 153-65.

8. Mutneja P, Dhawan P, Raina A, Sharma G. Menopause and the oral cavity. Ind J Endocrinol Metab 2012; 16(4): 548-51.

9. Sambashivaiah SCS, Bilichodmath S, Subbaiah R. Desquamative Gingivitis as a Clinical Manifestation in Endocrinal Imbalance-A Case Report. Ind J Stomatol 2011; 2(1): 49-52.

10. Abraham A, Pullishery F. The Effect of Menopause on the Periodontium-A Review. J Dent Med Sci 2015; 14(4): 79-82.

11. Hariri R, Alzoubi EE. Oral manifestations of menopause. J Dent Health Oral Disord Ther 2017; 7(4): 306-309.

12. Johnston BD, Ward WE. The ovariectomized rat as a model for studying alveolar bone loss in postmenopausal women. Biomed Res Int 2015; 2015(1): 635023-26.

13. Amadei SU, Souza DM, Brandao AA, Rocha RF. Influence of different durations of estrogen deficiency on alveolar bone loss in rats. Braz Oral Res 2011; 25(6): 538-43.

14. Anbinder AL, Prado Mde A, Spalding M, Balducci I. Estrogen deficiency and periodontal condition in rats: a radiographic and macroscopic study. Braz Dent J 2006; 17(3): 201-207.

15. Orrico SR, Goncalves D, Galeazzi ST, Giro G, Takayama L, Pereira RM. The influence of loss of bone mass on induced periodontal disease: a radiographic and densitometric study of female rats. J Periodontol 2005; 76(9): 1436-42.

16. Marques MR, da Silva MA, Manzi FR, Cesar-Neto JB, Nociti FH, Barros SP. Effect of intermittent PTH administration in the periodontitis-associated bone loss in ovariectomized rats. Arch Oral Biol 2005; 50(4): 421-29.

17. $\mathrm{Xu} \mathrm{XC}$, Chen $\mathrm{H}$, Zhang $X$, Zhai ZJ, Liu $X Q$, Zheng $X Y$, et al. Effects of oestrogen deficiency on the alveolar bone of rats with experimental periodontitis. Mol Med Rep 2015; 12(3): 3494-502.

18. Duarte PM, Goncalves PF, Sallum AW, Sallum EA, Casati MZ, Humberto Nociti F. Effect of an estrogen-deficient state and its therapy on bone loss resulting from an experimental periodontitis in rats. J Periodontal Res 2004; 39(2): 107-10.

19. Anbinder AL, Moraes RM, Lima GMG, Oliveira FE, Campos DRC, Rossoni RD, et al. Periodontal disease exacerbates systemic ovariectomy-induced bone loss in mice. Bone 2016; 83(1): 241-47.

20. Dai J, Ma Y, Shi M, Cao Z, Zhang Y, Miron RJ. Initial changes in alveolar bone volume for sham-operated and ovariectomized rats in ligature-induced experimental periodontitis. Clin Oral Investig 2016; 20(3): 581-88.

21. Liu Z, Liu L, Kang C, Xie Q, Zhang B, Li Y. Effects of estrogen deficiency on microstructural changes in rat alveolar bone proper and periodontal ligament. Mol Med Rep 2015; 12(3): 3508-14.

22. Romualdo PC, Lucisano MP, Paula-Silva FWG, Leoni GB, SousaNeto. Ovariectomy exacerbates apical period-ontitis in rats with an increase in expression of proinflammatory cytokines and matrix metalloproteinases. J Endod 2018; 44(5): 780-85.

23. Andriy S, Inna HH. Periodontal status of patients in different periods after total ovariectomy. Modern Science-Moderni Veda 2019; 6(2): 105-10.

24. Shapiro LF, Freeman K. The relationship between estrogen, estrogen receptors and periodontal disease in adult women: a review of the literature. NY State Dent J 2014; 80(3): 30-34. 